Research Article

\title{
Inverse Estimation of Temperature Profiles in Landfills Using Heat Recovery Fluids Measurements
}

\author{
C. Solisio, A. P. Reverberi, A. Del Borghi, and V. G. Dovi' \\ Department of Chemical and Process Engineering "G. B. Bonino", University of Genova, \\ Via Opera Pia 15, 16145 Genova, Italy \\ Correspondence should be addressed to V. G. Dovi', dovi@istic.unige.it
}

Received 8 December 2011; Accepted 4 March 2012

Academic Editor: Fu-Yun Zhao

Copyright (C) 2012 C. Solisio et al. This is an open access article distributed under the Creative Commons Attribution License, which permits unrestricted use, distribution, and reproduction in any medium, provided the original work is properly cited.

\begin{abstract}
In addition to leachate and gas emission analysis, temperature variations in municipal solid waste landfills are routinely monitored for safety and health reasons, such as the increased production of biogas or the danger of spontaneous combustion phenomena if the temperature exceeds 70 $75^{\circ} \mathrm{C}$. The increasing constraints on greenhouse gas emissions and the convenience of fuel and heat recovery have helped develop a global approach to landfills' operation and maintenance, generally referred to as bioreactor landfill management. The heat recovery piping we are presently designing can be a significant part of this approach. The heat gained by a fluid circulated in a closed network through the landfill is transferred to an external heat exchanger or used directly as warm water. Additionally, it can help reduce landfill temperature levels and control biogas generation. Since the pipes diameter is large enough to allow for a radial temperature gradient, this information can be used for an inverse estimation of the temperature profile in the landfill which constitutes the boundary conditions of the resulting heat transfer problem. In this paper, we describe an algorithm for regularising the resulting ill-posed free boundary estimation problem using sampled data of the heat recovery fluid on exiting the landfill.
\end{abstract}

\section{Introduction}

A new approach to landfills' operation and maintenance, generally referred to as "bioreactor landfill management," is expected to reduce the amount of leachate and to increase the production of biogas, while reducing the amount of necessary land.

For the achievement of this result, leachate and gas emissions of municipal solid waste landfills are accurately analysed, whereas temperature variations are routinely monitored for safety and health reasons, such as the increased production of biogas or the danger of spontaneous combustion phenomena if the temperature exceeds $70-75^{\circ} \mathrm{C}$ [1].

Significant amounts of heat are produced in municipal landfills due to decomposition of organic solid wastes. While leachate and gas emissions have been the object of several 
studies [2-4], less information is available on heat recovery technologies. However, they could be a significant part in the general bioreactor landfill management, as the heat gained by a fluid circulated in a closed network through the landfill can be transferred to an external heat exchanger or used directly as warm water adding to the overall energy saving strategy. Additionally, the heat removed can contribute to a reduction of temperature levels inside the landfill and to an alleviation of spontaneous combustion risks.

Since the diameter of the pipes carrying the heat recovery fluid is large enough to allow for a radial temperature gradient, this information can be used for an inverse estimation of the temperature profile in the landfill which constitutes the boundary conditions of the resulting heat transfer problem. This information can be combined with the measurements provided by in situ sensors (including thermocouples, thermistors, and vibrating wire piezometers) which have to withstand a corrosive aggressive environment and strong mechanical stresses. The simultaneous use of direct measurements provided by sensors and of estimates resulting from inverse temperature profile reconstructions can improve the overall thermal control in landfills.

The inverse estimation of the boundary conditions of a partial differential equations is known to be an ill-posed problem which requires suitable regularisation techniques to obtain stable and physically acceptable solutions.

In this paper, we employ a piecewise linear approximation to the unknown profile combined with a traditional Tikhonov regularisation technique [5]. The piecewise linear approximation of a boundary condition was first employed by the authors in the estimation of diffusivities in solids [6], where it was used in conjunction with separable least squares. In this paper, the method is extended to the thermal energy transfer of a fluid in stationary motion.

Exact simulated data are generated from an assumed smooth temperature profile with a spike to indicate the presence of a hot spot which might turn into a landfill fire. A Gaussian distributed error with zero mean and a standard deviation equal to the measurement precision is added to the exact data.

The reconstructions obtained by using different values for the number of intervals of the piecewise linear approximation and Tikhonov's regularisation parameter show the usefulness and the limitations of the method developed.

\section{Theoretical Development}

The temperature profile inside the pipes is determined by the transfer of heat from the soil to the fluid flowing in the pipes.

Assuming radial symmetry about the axis of the pipe and a flat velocity distribution across the section of pipe (which is a reasonable assumption due to the turbulent motion of the fluid), the energy balance can be written as [7]

$$
\begin{gathered}
\rho c_{p} u \frac{\partial T}{\partial x}=K \frac{1}{r} \frac{\partial}{\partial r}\left(r \frac{\partial T}{\partial r}\right), \\
\left(\frac{\partial T}{\partial r}\right)_{r=0}=0, \\
T(0, r)=T_{0}, \\
-K\left(\frac{\partial T}{\partial r}\right)_{r=R}=H[T(x, R)-\varphi(x)],
\end{gathered}
$$


where $\rho$ is the density, $c_{P}$ is the specific heat at constant pressure, $u$ is the speed, and $K$ is the conductivity of the fluid. $R$ is the radius of the pipe, and $x$ indicates the direction of motion. $H$ is the heat transfer coefficient from the soil to the fluid (including wall resistance), and $\varphi(x)$ is the unknown temperature profile of the soil.

Setting $k=K /\left(\begin{array}{lll}\rho & c_{P} & u\end{array}\right),(2.1)$ becomes

$$
\frac{\partial T}{\partial x}=k \frac{1}{r} \frac{\partial}{\partial r}\left(r \frac{\partial T}{\partial r}\right)
$$

This equation is formally equal to the transient heat conduction in an infinite circular cylinder [8].

The general analytical solution of (2.1)-(2.4) can be determined by applying Duhamel's principle [9] to the particular solution obtained assuming a constant temperature profile along the $x$ direction.

Indeed, if the soil temperature profile is constant along $x\left(\varphi(x)=T^{*}\right)$, the analytical solution is provided by the infinite series [8]

$$
\frac{\left(T-T_{0}\right)}{\left(T^{*}-T_{0}\right)}=1-2 A \sum_{n=1}^{\infty} e^{-\left(\beta_{n}^{2} / R^{2}\right) k x} \frac{J_{0}\left((r / R) \beta_{n}\right)}{\left(\beta_{n}^{2}+A^{2}\right) J_{0}\left(\beta_{n}\right)}
$$

where $A=R H, J_{0}$ is the Bessel function of order zero, and $\beta_{n}$ are the roots of

$$
\beta J_{1}(\beta)=A J_{0}(\beta)
$$

Combining this equation with Duhamel's principle, we obtain the general solution

$$
T(r, x)-T_{0}=\frac{2 A k}{R^{2}} \sum_{n=1}^{\infty} \beta_{n} e^{-\left(\beta_{n}^{2} / R^{2}\right) k x} \frac{J_{0}\left((r / R) \beta_{n}\right)}{\left(\beta_{n}^{2}+A^{2}\right) J_{0}\left(\beta_{n}\right)} \int_{0}^{x} e^{\left(\beta_{n}^{2} / R^{2}\right) k \lambda} \varphi(\lambda) d \lambda
$$

Thus, if the temperature of the fluid $T(r, x)$ is measured at the exit of the landfill $(x=L)$, the unknown soil temperature profile $\varphi(x)$ can be estimated.

Following the method developed in [6], let us approximate the profile by a continuous piecewise function $\psi(x)$ in the range $[0, L]$ divided into $M$ equal intervals of width $h=L / M$,

$$
\psi(x)=T_{0}+h \sum_{i=1}^{k-1} c_{i}+c_{k}\left(x-x_{k-1}\right), \quad x_{k-1} \leq x \leq x_{k},
$$

where $c_{k}$ is the angular coefficient of the linear approximation in the interval $x_{k-1} \leq x \leq x_{k}$. 
The integrals in the infinite series can now be approximated by

$$
\begin{aligned}
\int_{0}^{x} e^{\left(\beta_{n}^{2} / R^{2}\right) k \lambda} \phi(\lambda) d \lambda= & \int_{0}^{x_{1}} e^{\left(\beta_{n}^{2} / R^{2}\right) k \lambda}\left(T_{0}+c_{1} \lambda\right) d \lambda+\int_{x_{1}}^{x_{2}} e^{\left(\beta_{n}^{2} / R^{2}\right) k \lambda}\left[T_{0}+h c_{1}+c_{2}\left(\lambda-x_{1}\right)\right] d \lambda+\cdots \\
& +\int_{x_{k-1}}^{x} e^{\left(\beta_{n}^{2} / R^{2}\right) k \lambda}\left[T_{0}+h \sum_{i=1}^{k-1} c_{i}+c_{k}\left(\lambda-x_{k-1}\right)\right] d \lambda, \quad x_{k-1} \leq x \leq x_{k}
\end{aligned}
$$

which can be rewritten as

$$
\begin{aligned}
\int_{0}^{x} e^{\left(\beta_{n}^{2} / R^{2}\right) k \lambda} \phi(\lambda) d \lambda= & T_{0}\left\{\int_{0}^{x_{1}} e^{\left(\beta_{n}^{2} / R^{2}\right) k \lambda} d \lambda+\int_{x_{1}}^{x_{2}} e^{\left(\beta_{n}^{2} / R^{2}\right) k \lambda} d \lambda+\cdots+\int_{x_{k-1}}^{x} e^{\left(\beta_{n}^{2} / R^{2}\right) k \lambda} d \lambda\right\} \\
& +c_{1}\left\{\int_{0}^{x_{1}} \lambda e^{\left(\beta_{n}^{2} / R^{2}\right) k \lambda} d \lambda+h \int_{x_{1}}^{x_{2}} e^{\left(\beta_{n}^{2} / R^{2}\right) k \lambda} d \lambda\right. \\
& \left.+\cdots+h \int_{x_{k-1}}^{x} e^{\left(\beta_{n}^{2} / R^{2}\right) k \lambda} d \lambda\right\} \\
& +c_{2}\left\{\int_{x_{1}}^{x_{2}}\left(\lambda-x_{1}\right) e^{\left(\beta_{n}^{2} / R^{2}\right) k \lambda} d \lambda+h \int_{x_{2}}^{x_{3}} e^{\left(\beta_{n}^{2} / R^{2}\right) k \lambda} d \lambda\right. \\
& \left.+\cdots+h \int_{x_{k-1}}^{x} e^{\left(\beta_{n}^{2} / R^{2}\right) k \lambda} d \lambda\right\} \\
& +c_{3}\left\{\int_{x_{2}}^{x_{3}}\left(\lambda-x_{2}\right) e^{\left(\beta_{n}^{2} / R^{2}\right) k \lambda} d \lambda+h \int_{x_{3}}^{x_{4}} e^{\left(\beta_{n}^{2} / R^{2}\right) k \lambda} d \lambda\right. \\
& \left.+\cdots+h \int_{x_{k-1}}^{x} e^{\left(\beta_{n}^{2} / R^{2}\right) k \lambda} d \lambda\right\} \\
& +\cdots \\
& +c_{2}\left\{\frac{R^{2}}{\beta_{n}^{2} k}\left(x_{2} e^{\left(\beta_{n}^{2} / R^{2}\right) k x_{2}}-x_{1} e^{\left(\beta_{n}^{2} / R^{2}\right) k x_{1}}\right)\right. \\
+ & \frac{T_{0} R^{2}}{k \beta_{n}^{2}}\left\{e^{\left(\beta_{n}^{2} / R^{2}\right) k x}-1\right\} \\
& \left.+\frac{h R^{2}}{\beta_{n}^{2} k}\left(e^{\left(\beta_{n}^{2} / R^{2}\right) k x}-e^{\left(\beta_{n}^{2} / R^{2}\right) k x_{1}}\right)\right\} \\
&
\end{aligned}
$$


Journal of Applied Mathematics

$$
\begin{aligned}
& -\left(\frac{R^{2}}{\beta_{n}^{2} k}\right)^{2}\left(e^{\left(\beta_{n}^{2} / R^{2}\right) k x_{2}}-e^{\left(\beta_{n}^{2} / R^{2}\right) k x_{1}}\right) \\
& +\frac{h R^{2}}{\beta_{n}^{2} k}\left(e^{\left(\beta_{n}^{2} / R^{2}\right) k x}-e^{\left(\beta_{n}^{2} / R^{2}\right) k x_{2}}\right) \\
& \left.\quad-\frac{x_{1} R^{2}}{\beta_{n}^{2} k}\left(e^{\left(\beta_{n}^{2} / R^{2}\right) k x_{2}}-e^{\left(\beta_{n}^{2} / R^{2}\right) k x_{1}}\right)\right\} \\
& +\cdots \quad c_{k}\left\{\frac{R^{2}}{\beta_{n}^{2} k}\left(x e^{\left(\beta_{n}^{2} / R^{2}\right) k x}-x_{k-1} e^{\left(\beta_{n}^{2} / R^{2}\right) k x_{k-1}}\right)\right. \\
& \quad-\left(\frac{R^{2}}{\beta_{n}^{2} k}\right)^{2}\left(e^{\left(\beta_{n}^{2} / R^{2}\right) k x}-e^{\left(\beta_{n}^{2} / R^{2}\right) k x_{k-1}}\right) \\
& \left.-\frac{x_{k-1} R^{2}}{\beta_{n}^{2} k}\left(e^{\left(\beta_{n}^{2} / R^{2}\right) k x}-e^{\left(\beta_{n}^{2} / R^{2}\right) k x_{k-1}}\right)\right\}, \quad x_{k-1} \leq x \leq x_{k} .
\end{aligned}
$$

Thus,

$$
\int_{0}^{x} e^{\left(\beta_{n}^{2} / R^{2}\right) k \lambda} \phi(\lambda) d \lambda=P_{n}+\sum_{i=1}^{k} c_{i} R_{\mathrm{in}}, \quad x_{k-1} \leq x \leq x_{k}
$$

where $P_{n}$ and $R_{\text {in }}$ can be evaluated by comparison.

We can now rewrite (2.8) as

$$
T(r, x)-T_{0}=\frac{2 A k}{R^{2}} \sum_{n=1}^{\infty} \beta_{n} e^{-\left(\beta_{n}^{2} / R^{2}\right) k x} \frac{J_{0}\left((r / R) \beta_{n}\right)}{\left(\beta_{n}^{2}+A^{2}\right) J_{0}\left(\beta_{n}\right)}\left(P_{n}+\sum_{i=1}^{k} c_{i} R_{\mathrm{in}}\right), \quad x_{k-1} \leq x \leq x_{k} .
$$

In order to estimate the value of $N$ such that

$$
\begin{aligned}
& \frac{2 A k}{R^{2}} \sum_{n=1}^{N} \beta_{n} e^{-\left(\beta_{n}^{2} / R^{2}\right) k x} \frac{J_{0}\left((r / R) \beta_{n}\right)}{\left(\beta_{n}^{2}+A^{2}\right) J_{0}\left(\beta_{n}\right)}\left(P_{n}+\sum_{i=1}^{k} c_{i} R_{\mathrm{in}}\right) \\
& \approx \frac{2 A k}{R^{2}} \sum_{n=1}^{\infty} \beta_{n} e^{-\left(\beta_{n}^{2} / R^{2}\right) k x} \frac{J_{0}\left((r / R) \beta_{n}\right)}{\left(\beta_{n}^{2}+A^{2}\right) J_{0}\left(\beta_{n}\right)}\left(P_{n}+\sum_{i=1}^{k} c_{i} R_{\mathrm{in}}\right)
\end{aligned}
$$

to any predefined accuracy, let us analyse first the infinite series

$$
\frac{2 A k}{R^{2}} \sum_{n=1}^{\infty} \beta_{n} e^{-\left(\beta_{n}^{2} / R^{2}\right) k x} \frac{J_{0}\left((r / R) \beta_{n}\right)}{\left(\beta_{n}^{2}+A^{2}\right) J_{0}\left(\beta_{n}\right)} P_{n}
$$


where

$$
\begin{gathered}
P_{n}=\frac{T_{0} R^{2}}{k \beta_{n}^{2}}\left\{e^{\left(\beta_{n}^{2} / R^{2}\right) k x}-1\right\}, \\
\frac{2 A k}{R^{2}} \sum_{n=1}^{\infty} \beta_{n} e^{-\left(\beta_{n}^{2} / R^{2}\right) k x} \frac{J_{0}\left((r / R) \beta_{n}\right)}{\left(\beta_{n}^{2}+A^{2}\right) J_{0}\left(\beta_{n}\right)}\left[\frac{T_{0} R^{2}}{k \beta_{n}^{2}}\left\{e^{\left(\beta_{n}^{2} / R^{2}\right) k x}-1\right\}\right] \\
=2 A T_{0}\left\{\sum_{n=1}^{\infty} \frac{1}{\beta_{n}} \frac{J_{0}\left((r / R) \beta_{n}\right)}{\left(\beta_{n}^{2}+A^{2}\right) J_{0}\left(\beta_{n}\right)}+\sum_{n=1}^{\infty} \frac{e^{-\left(\beta_{n}^{2} / R^{2}\right) k x}}{\beta_{n}} \frac{J_{0}\left((r / R) \beta_{n}\right)}{\left(\beta_{n}^{2}+A^{2}\right) J_{0}\left(\beta_{n}\right)}\right\} .
\end{gathered}
$$

Let us consider the first series in (2.16).

Since $\beta_{n}>\beta_{1}+3(n-1)$,

$$
\begin{aligned}
\sum_{n=N+1}^{\infty} \frac{1}{\beta_{n}} \frac{J_{0}\left((r / R) \beta_{n}\right)}{\left(\beta_{n}^{2}+A^{2}\right) J_{0}\left(\beta_{n}\right)} & <\sum_{n=N+1}^{\infty} \frac{1}{\beta_{1}+3(n-1)} \frac{J_{0}\left((r / R) \beta_{n}\right)}{\left(\beta_{n}^{2}+A^{2}\right) J_{0}\left(\beta_{n}\right)} \\
& <\frac{1}{\beta_{1}+3 N} \sum_{n=N+1}^{\infty} \frac{J_{0}\left((r / R) \beta_{n}\right)}{\left(\beta_{n}^{2}+A^{2}\right) J_{0}\left(\beta_{n}\right)} .
\end{aligned}
$$

Setting $x=0$ in (2.8), we can verify that for all $r / R$,

$$
\sum_{n=1}^{\infty} \frac{J_{0}\left((r / R) \beta_{n}\right)}{\left(\beta_{n}^{2}+A^{2}\right) J_{0}\left(\beta_{n}\right)}=\frac{1}{2 A} .
$$

Consequently,

$$
\sum_{n=N+1}^{\infty} \frac{J_{0}\left((r / R) \beta_{n}\right)}{\left(\beta_{n}^{2}+A^{2}\right) J_{0}\left(\beta_{n}\right)}=\frac{1}{2 A}-\sum_{n=1}^{N} \frac{J_{0}\left((r / R) \beta_{n}\right)}{\left(\beta_{n}^{2}+A^{2}\right) J_{0}\left(\beta_{n}\right)}
$$

Thus, for any $N$, we can verify whether

$$
\sum_{n=N+1}^{\infty} \frac{1}{\beta_{n}} \frac{J_{0}\left((r / R) \beta_{n}\right)}{\left(\beta_{n}^{2}+A^{2}\right) J_{0}\left(\beta_{n}\right)}<\frac{1}{\beta_{1}+3 N}\left[\frac{1}{2 A}-\sum_{n=1}^{N} \frac{J_{0}\left((r / R) \beta_{n}\right)}{\left(\beta_{n}^{2}+A^{2}\right) J_{0}\left(\beta_{n}\right)}\right]<\varepsilon,
$$

where $\varepsilon$ is any predetermined accuracy.

The same truncation index $N$ can be used to estimate a sufficient number of terms in the second series.

Indeed, let us rewrite

$$
\begin{aligned}
\frac{2 A k}{R^{2}} & \sum_{n=1}^{\infty} \beta_{n} e^{-\left(\beta_{n}^{2} / R^{2}\right) k x} \frac{J_{0}\left((r / R) \beta_{n}\right)}{\left(\beta_{n}^{2}+A^{2}\right) J_{0}\left(\beta_{n}\right)}\left[\frac{T_{0} R^{2}}{k \beta_{n}^{2}}\left\{e^{\left(\beta_{n}^{2} / R^{2}\right) k x}-1\right\}\right] \\
& =2 A T_{0}\left\{\sum_{n=1}^{\infty} a_{n}+\sum_{n=1}^{\infty} a_{n} e^{-\left(\beta_{n}^{2} / R^{2}\right) k x}\right\},
\end{aligned}
$$


and let $N$ be the index such that

$$
\frac{\sum_{n=N+1}^{\infty} a_{n}}{\sum_{n=1}^{N} a_{n}} \leq \varepsilon
$$

Since

$$
\begin{gathered}
\sum_{n=1}^{N} a_{n} e^{-\left(\beta_{n}^{2} / R^{2}\right) k x}>e^{-\left(\beta_{N}^{2} / R^{2}\right) k x} \sum_{n=1}^{N} a_{n} \\
\sum_{n=N+1}^{\infty} a_{n} e^{-\left(\beta_{n}^{2} / R^{2}\right) k x}<e^{-\left(\beta_{N}^{2} / R^{2}\right) k x} \sum_{n=N+1}^{\infty} a_{n} \\
\frac{\sum_{n=N+1}^{\infty} a_{n} e^{-\left(\beta_{n}^{2} / R^{2}\right) k x}}{\sum_{n=1}^{N} a_{n} e^{-\left(\beta_{n}^{2} / R^{2}\right) k x}}<\frac{e^{-\left(\beta_{N}^{2} / R^{2}\right) k x} \sum_{n=N+1}^{\infty} a_{n}}{e^{-\left(\beta_{N}^{2} / R^{2}\right) k x} \sum_{n=1}^{N} a_{n}}<\varepsilon,
\end{gathered}
$$

consequently $N$ is a conservative estimate also for the second series.

It can be shown that using a similar procedure, the same truncation index can be used also for the series

$$
\sum_{n=1}^{\infty} c_{i} R_{\mathrm{in}} \beta_{n} e^{-\left(\beta_{n}^{2} / R^{2}\right) k x} \frac{J_{0}\left((r / R) \beta_{n}\right)}{\left(\beta_{n}^{2}+A^{2}\right) J_{0}\left(\beta_{n}\right)} .
$$

All the previous algebraic expressions can be combined to provide the final relationship between the radial distribution of temperatures in a section of the pipe at a distance $L$ from the point where heat transfer with the soil starts

$$
\begin{gathered}
T(r, L)=T_{0}+\omega(r)+\sum_{i=1}^{M} c_{i} \xi_{i}(r), \\
\omega(r)=\frac{2 A k}{R^{2}} \sum_{n=1}^{N} \beta_{n} P_{n}(L) e^{-\left(\beta_{n}^{2} / R^{2}\right) k L} \frac{J_{0}\left((r / R) \beta_{n}\right)}{\left(\beta_{n}^{2}+A^{2}\right) J_{0}\left(\beta_{n}\right)}, \\
\xi_{i}(r)=\frac{2 A k}{R^{2}} \sum_{n=1}^{N} \beta_{n} R_{\mathrm{in}}(L) e^{-\left(\beta_{n}^{2} / R^{2}\right) k L} \frac{J_{0}\left((r / R) \beta_{n}\right)}{\left(\beta_{n}^{2}+A^{2}\right) J_{0}\left(\beta_{n}\right)} .
\end{gathered}
$$

For each $r$, the value of $N$ is determined by the condition

$$
\frac{1}{\beta_{1}+3 N}\left[\frac{1}{2 A}-\sum_{n=1}^{N} \frac{J_{0}\left((r / R) \beta_{n}\right)}{\left(\beta_{n}^{2}+A^{2}\right) J_{0}\left(\beta_{n}\right)}\right] \approx \varepsilon \sum_{n=1}^{N} \frac{1}{\beta_{n}} \frac{J_{0}\left((r / R) \beta_{n}\right)}{\left(\beta_{n}^{2}+A^{2}\right) J_{0}\left(\beta_{n}\right)}
$$

where $\varepsilon$ is typically $10^{-6}$. 
For any data set,

$$
y_{j}=T\left(r_{j}, L\right)-T_{0}-\omega\left(r_{j}\right),
$$

we can estimate the slopes of the piecewise linear temperature distribution of the soil from the system of linear equations

$$
y_{j}=\sum_{i=1}^{M} \xi_{i j} c_{i}
$$

The usual least-squares estimation procedure provides the well-known relationship

$$
\widehat{\mathbf{c}}=\left(\Gamma^{*} \Gamma\right)^{-1} \Gamma^{*} \mathbf{y},
$$

where $\widehat{\mathbf{c}}$ is the vector of the estimated slopes and $\Gamma=\left\|\xi_{i j}\right\|$.

Since this estimation problem is an ill-posed problem, the accuracy of the estimates $\widehat{c}$ does not depend continuously on the experimental errors on the data.

The results obtained applying Tikhonov's regularisation method to this problem will be discussed in the next sections using simulated data generated by a smooth temperature profile with a spike representing a hot spot in the landfill.

\section{Regularisation Technique}

Reducing the unknown soil temperature function to a finite-dimensional vector $\widehat{c}$ changes the theoretically ill-posed problem into a numerically ill-conditioned one, reflected by near singularity of the matrix $\Gamma^{*} \Gamma$.

Thus, the most frequently used regularisation method consists in the modification and/or elimination of the eigenvalues of $\Gamma^{*} \Gamma$ that are too small.

A possible way to do this is reducing the dimension of the matrix (which in our case implies reducing the number of intervals over which the temperature of the soil is approximately linear). For this approach to be effective, the dimension of the vector $\widehat{\mathbf{c}}$ has frequently to be reduced so severely as to result in an unacceptably poor reconstruction.

A more efficient technique is the one proposed by Tikhonov and Arsenine [5] and based on the statistical properties of the experimental errors on the set of measured variables y.

To illustrate it, let us suppose that measured $\mathbf{y}^{\prime}$ and exact (unknown) values $\mathbf{y}$ of the experimental data are related by the relationship

$$
\left\|\mathbf{y}-\mathbf{y}^{\prime}\right\|^{2} \leq \delta
$$

where $\delta$ is determined by the variance of the statistical distribution of the experimental error.

Tikhonov's regularisation method consists in replacing the original minimisation problem

$$
\left\|\Gamma \widehat{\mathbf{c}}-\mathbf{y}^{\prime}\right\|^{2}=\min ,
$$


Table 1

\begin{tabular}{lcc}
\hline Parameter & Value & Symbol \\
\hline Fluid speed & $1 \mathrm{~m} / \mathrm{s}$ & $u$ \\
Pipe diameter & $0.4 \mathrm{~m}$ & $2 R$ \\
Fluid density & $1000 \mathrm{Kg} / \mathrm{m}^{3}$ & $\rho$ \\
Fluid conductivity & $0.6397 \mathrm{~W} /\left(\mathrm{s} \mathrm{m}^{\circ} \mathrm{K}\right)$ & $\mathrm{K}$ \\
Heat transfer coefficient* & $90.36 \mathrm{~J} /\left(\mathrm{s} \mathrm{m}^{2}{ }^{\circ} \mathrm{K}\right)$ & $H$ \\
\hline
\end{tabular}

"Data taken from [11] ("Ground Source Heat Pumps: Design of Geothermal Systems for Commercial and Institutional Buildings"), ASHRAE (2008).

with the minimisation problem

$$
\left\|\Gamma \widehat{\mathbf{c}}-\mathbf{y}^{\prime}\right\|^{2}+\alpha\|\widehat{\mathbf{c}}\|^{2}=\min
$$

where the positive constant $\alpha$ is chosen so that

$$
\left\|\Gamma \widehat{\mathbf{c}}-\mathbf{y}^{\prime}\right\|^{2}=\delta
$$

Combining (3.3) and (3.4) provides the reconstructed vector $\widehat{\mathbf{c}}$,

$$
\widehat{\mathbf{c}}=\left(\Gamma^{*} \Gamma+\alpha \mathbf{I}\right)^{-1} \Gamma^{*} \mathbf{y}^{\prime}
$$

with $\alpha$ being determined by Morozov's discrepancy method [10], that is, iteratively increasing the value of $\alpha$ until (3.4) is satisfied.

Clearly the eigenvalues of $\Gamma^{*} \Gamma$ are now increased by an amount $\alpha$, which being positive regularises the solution vector $\widehat{\mathbf{c}}$ at the price of smoothing it. The compromise between regularisation and accuracy is given by (3.4), which being rooted in statistics provides a theoretical framework for an optimal offset.

However, caution is warranted when considering automatic acceptance of the solution obtained. Indeed, the value of $\delta$ (related to the statistical distribution of the experimental error) might be only approximately known. Additionally, the variance of the sample can differ substantially from the theoretical one, thus leading to a biased value of $\delta$.

Therefore, solution vectors $\widehat{\mathbf{c}}$ obtained by letting $\alpha$ vary around the optimal Tikhonov's value (typically an order of magnitude above and below) should be examined for the detection of possible over- or undersmoothing effects.

\section{A Numerical Example}

Exact simulated data have been generated using a smooth parabolic temperature profile with a spike to indicate the presence of a hot spot which might turn into a landfill fire. The profile of the physical model is plotted in Figure 1. The geometrical and physical-chemical parameters used are reported in Table 1.

Since the fluid used is water, both fluid density and fluid conductivity can be estimated with a very high degree of accuracy. Consequently the first four parameters can be assumed to 


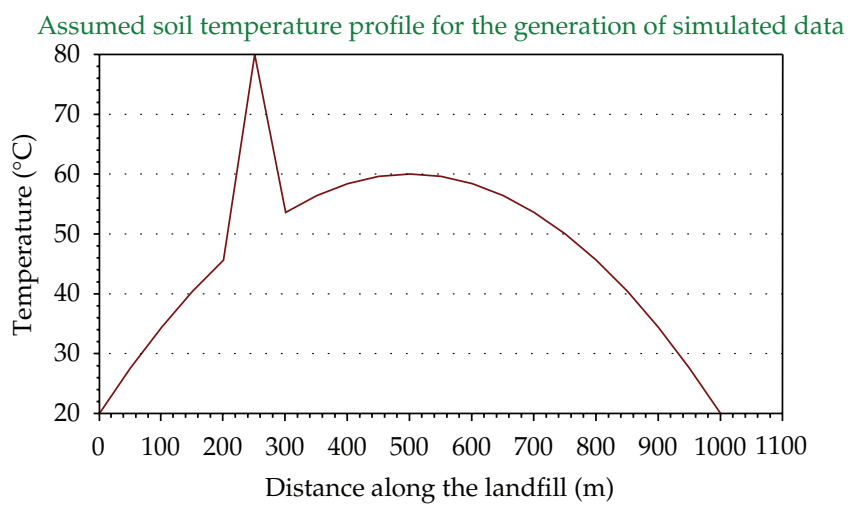

Figure 1

be accurately known. On the other hand, the heat transfer coefficient depends on the nature of soil. Since the goal of this paper is to show the reliability of the general procedure, the literature value (recommended by the American Society of Heating and Air-Conditioning for ordinary soil) has been used.

More accurate values (based on an experimental campaign) should be employed when real life cases are considered. Additionally, the uncertainties resulting from the standard deviation of the estimate of the heat transfer coefficient should be taken into account. To this purpose, a preliminary parameter sensitivity analysis is carried out in this paper by letting the parameter $H$ vary by $10 \%$ about the value recommended by the ASHRAE.

The computation of data was carried out using (2.8). The truncation index was evaluated using the procedure described previously, whereas the integrals were computed analytically.

A Gaussian distributed error with zero mean and a standard deviation equal to the measurement precision $\left(10^{-3 \circ} \mathrm{C}\right)$ was added to the exact simulated data.

The soil temperature profile was reconstructed using 10,12, 15, and 20 equally spaced intervals in each of which the temperature profile was assumed linear. The regularisation parameter was estimated using Tikhonov's stabilisation method based on the statistical assumptions.

The corresponding graphs are plotted in Figures 2, 3, 4, and 5, respectively.

In all reconstructions, there is no clear identification of a peak, rather a smooth "bump" appears in all reconstructions at approximately the right place. Thus, the number of intervals does not seem to significantly affect the reconstruction of the temperature profile.

On the other hand, if Tikhonov's parameter is reduced by a factor 2, the presence of a temperature peak at the right position is more clearly visible. The value of the peak is also estimated with good accuracy as shown in the 15-interval reconstruction plotted in Figure 6.

The increased accuracy in position and intensity of the peak value is offset by more marked oscillations, as could be expected owing to the reduction of the smoothing factor.

As expected, increasing Tikhonov's parameter by a factor 2 smooths the temperature profile further and blurs the shape of the peak, as shown in Figure 7.

In all reconstructions, there is a hint that a hot spot with a larger-than-expected temperature is present in the landfill. The position is also predicted with reasonable accuracy. Reducing the value of Tikhonov's parameter below its theoretically optimal value provides an excellent estimation of position and intensity of the temperature peak. Thus, combining 


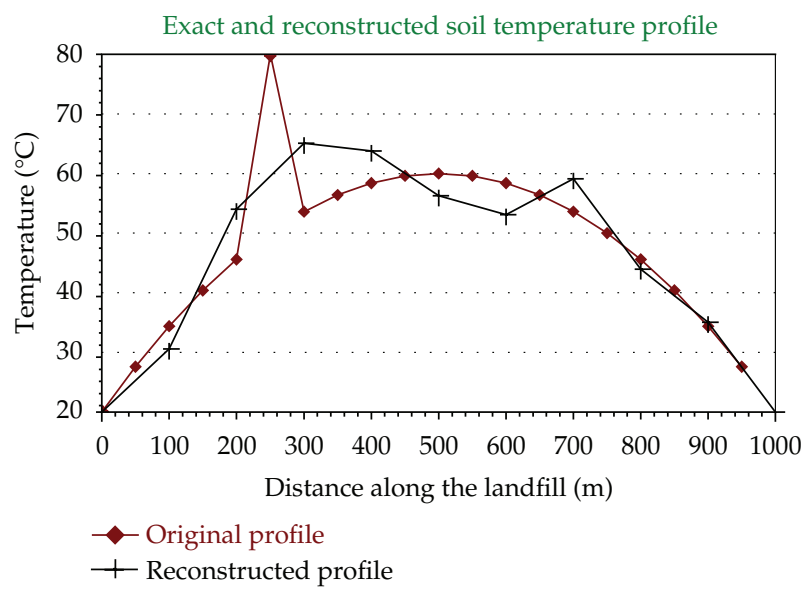

Figure 2: Ten intervals-Tikhonov's regularisation factor.

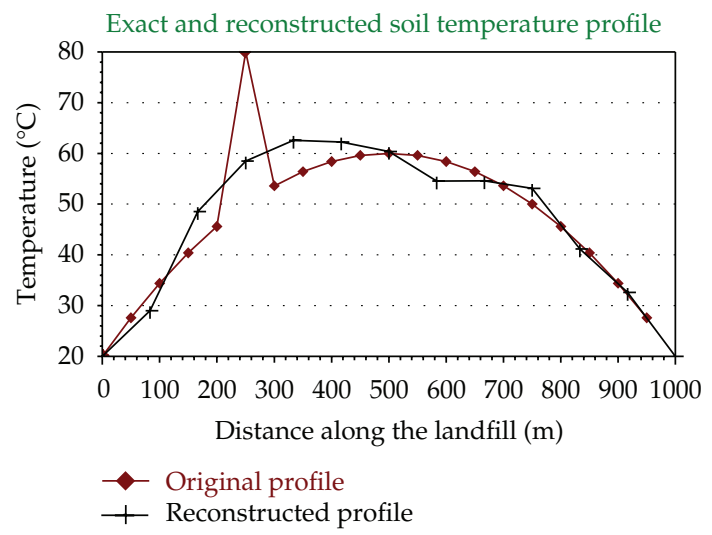

Figure 3: Twelve intervals-Tikhonov's regularisation factor.

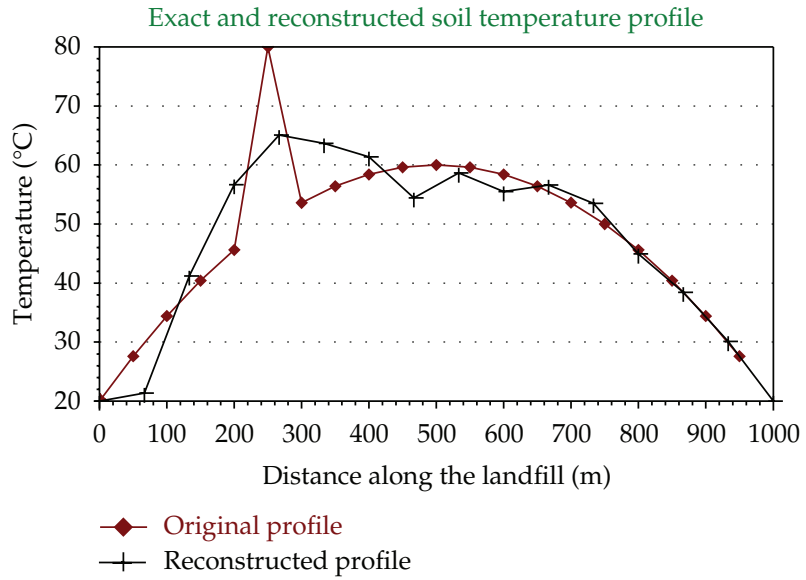

Figure 4: Fifteen intervals-Tikhonov's regularisation factor. 


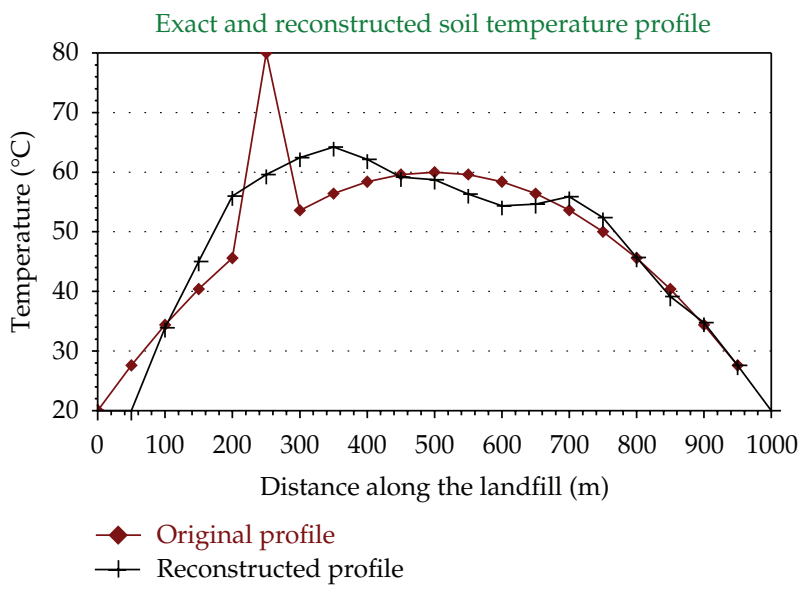

Figure 5: Twenty intervals-Tikhonov's regularisation factor.

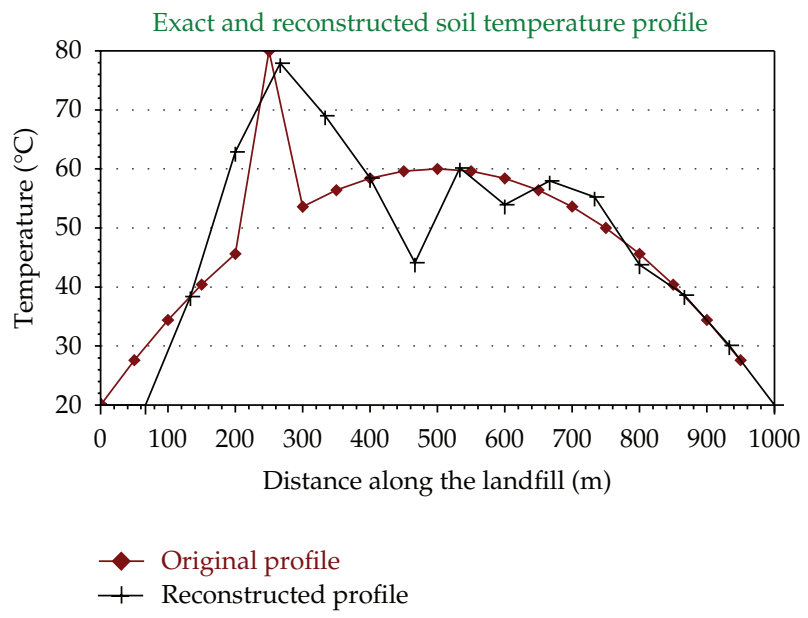

Figure 6: Fifteen intervals-Half Tikhonov's regularisation factor.

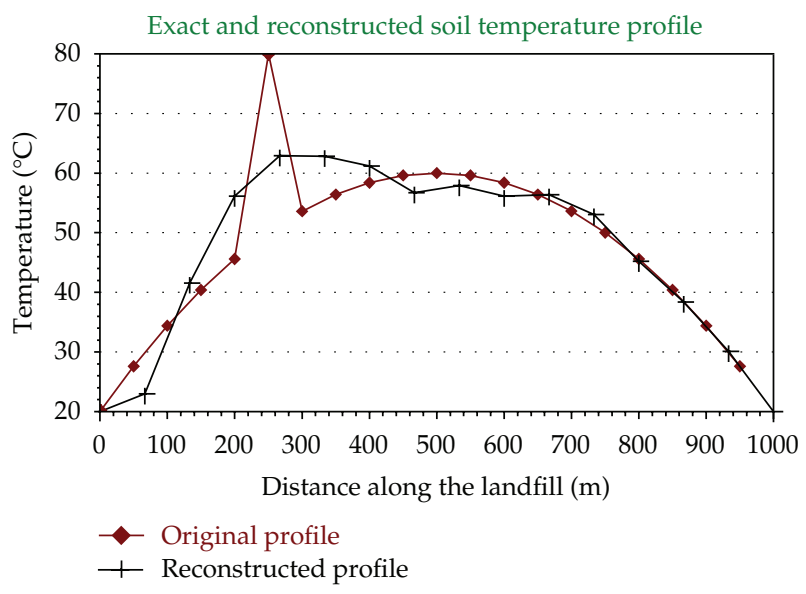

Figure 7: Fifteen intervals—double Tikhonov's regularisation factor. 


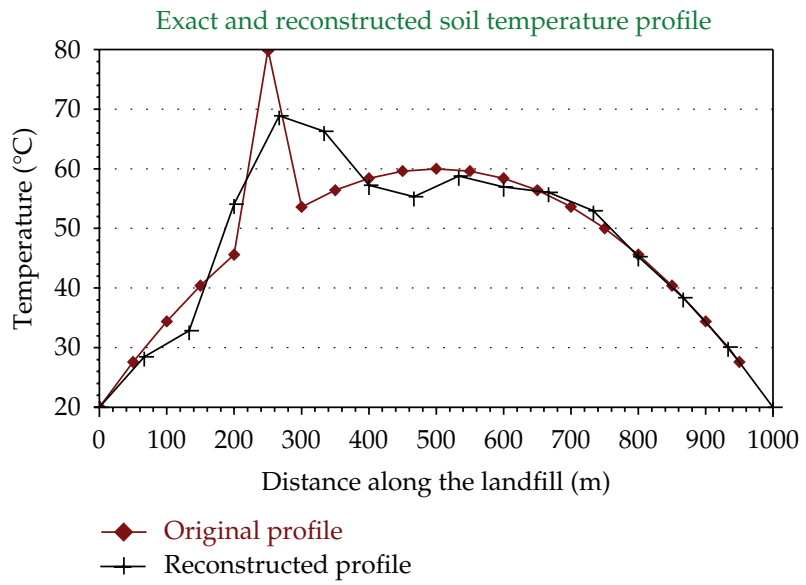

Figure 8: Fifteen intervals-Tikhonov's regularisation factor-assumed heat transfer coefficient equal to $90 \%$ of the value used to generate the simulated data.

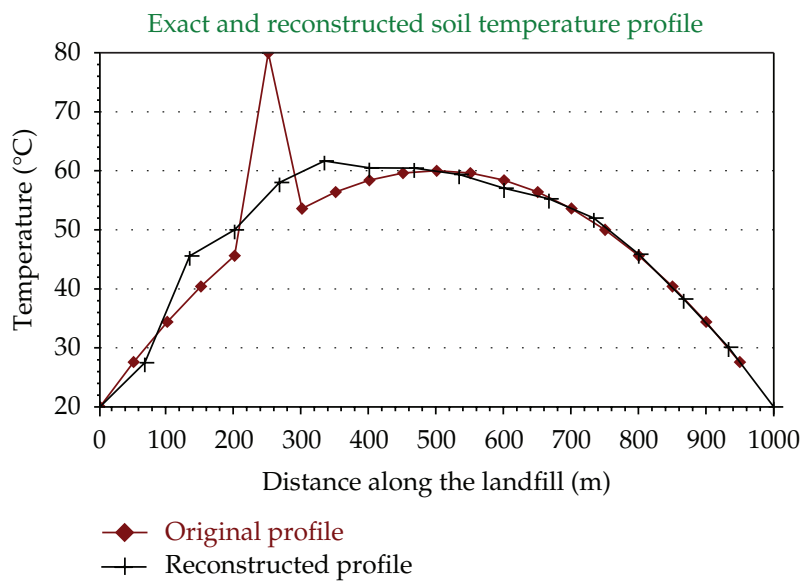

Figure 9: Fifteen intervals-Tikhonov's regularisation factor-assumed heat transfer coefficient equal to $110 \%$ of the value used to generate the simulated data.

Tikhonov's regularisation method with a strategy of exploring additional reconstructions by letting the regularisation parameter vary around its theoretical value provides valuable information for the overall monitoring process.

As mentioned before, the only parameter subject to a certain degree of uncertainty is the heat transfer coefficient. Even if good estimates can be obtained through a limited number of measurements in a well-designed experimental campaign, it is important to verify the consequences of the use of an incorrect heat transfer coefficient on the reconstruction of the temperature profile.

To this purpose, a simple sensitivity analysis has been performed by examining reconstructed profiles when the heat transfer coefficient employed in the regression analysis differs by $10 \%$ from the corresponding value used in the generation of the simulated data.

The heat transfer coefficient $H$ is directly proportional to the variable $A$. Furthermore, it follows from the relation $\beta J_{1}(\beta)=A J_{0}(\beta)$ that the variables $\beta_{n}$ are increasing functions of $A$. 
Thus, both $\omega$ and $\xi$ (and consequently the vector $y^{\prime}$ and the matrix $\Gamma$ ) are decreasing functions of the heat transfer coefficient used in the reconstruction. Therefore a higher value of $H$ is equivalent to an increased weight of the regularisation term in the minimisation problem (3.3) and consequently to a more marked smoothing effect.

This is confirmed by the two further reconstructions obtained using 15 equally spaced intervals, the theoretical Tikhonov's regularisation factor, and values of the heat transfer coefficient equal to $90 \%$ and $110 \%$ of the original value, respectively. As shown in Figures 8 and 9 , even a $10 \%$ deviation from the true value (which can be regarded as an upper estimate error) does not affect to any significant degree the analysis made.

Furthermore, it can prove difficult to distinguish between over- (under)smoothing due to larger- (smaller-) than-optimal value of Tikhonov's factor and a wrong estimate of the heat transfer coefficient. This can be regarded as an added reason for considering a reasonably wide range of values of this factor.

\section{Conclusions}

If and when the heat generated in a landfill is extracted by the circulation of a heat recovery fluid, some additional information on temperature distribution inside the landfill will be available as an added benefit.

The reconstruction technique described in this paper is not intended to replace the traditional monitoring, but rather to complement it, making it possible to reduce the number of sensors and/or to detect potential combustion phenomena at places where no sensor is installed. Furthermore, mobile temperature monitoring equipment can be more efficiently allocated following the indications provided by the implementation of the algorithm developed in this paper.

\section{Notation}

\section{Symbols}

$c_{i}$ : Slope of temperature in the interval $i\left({ }^{\circ} \mathrm{C} / \mathrm{m}\right)$

$c_{p}$ : Specific heat of heat recovery fluid

$h$ : Interval of linear dependance of temperature on distance $(\mathrm{m})$

$H$ : Soil heat transfer coefficient $\left(\mathrm{J} /\left(\mathrm{s} \cdot \mathrm{m}^{2} \cdot{ }^{\circ} \mathrm{K}\right)\right)$

$J_{0}$ : Bessel function of order zero

$J_{1}$ : Bessel function of order one

$\mathrm{K}$ : Heat conduction coefficient of heat recovery fluid $\left(\mathrm{W} /\left(\mathrm{s} \cdot \mathrm{m} \cdot{ }^{\circ} \mathrm{C}\right)\right)$

$L:$ Distance travelled by the fluid in the landfill (m)

$M$ : Number of equally spaced intervals in $L$

$N$ : Number of terms in the infinite series necessary to achieve a relative accuracy $\varepsilon$

$u$ : Speed of heat recovery fluid $(\mathrm{m} / \mathrm{s})$

$r$ : Radial variable in the pipe cross section $(\mathrm{m})$

$R$ : Pipe radius $(\mathrm{m})$

T: Temperature $\left({ }^{\circ} \mathrm{C}\right)$

$T_{0}$ : Ambient temperature $\left({ }^{\circ} \mathrm{C}\right)$

$x$ : Variable in the direction of fluid motion (m)

$y$ : Experimental data set 
$\alpha$ : Tikhonov's regularisation parameter

$\varepsilon$ : Relative accuracy in the evaluation of series

$\lambda$ : Dummy integration variable

$\rho$ : Density of heat recovery fluid $\left(\mathrm{Kg} / \mathrm{m}^{3}\right)$

$\varphi$ : Unknown exact soil temperature profile $\left({ }^{\circ} \mathrm{C}\right)$.

Symbols Defined by Equations

A: $\quad R \cdot H$

$k: \quad K /\left(\rho \cdot c_{p} \cdot u\right)$

$P_{n}, R_{\text {in }}$ : Defined by the equation $\int_{0}^{L} e^{\left(\beta_{n}^{2} / R^{2}\right) k \lambda} \varphi(\lambda) d \lambda=P_{n}+\sum_{i=1}^{M} c_{i} R_{\text {in }}$

$\beta_{n}: \quad n$th root of the equation $\beta J_{1}(\beta)=A J_{0}(\beta)$

$\Gamma$ : $\quad$ Matrix whose elements are $\xi_{i j}$

$\delta$ : $\quad$ Parameter determined by the variance of the statistical distribution of the experimental error

$\xi, \omega: \quad$ Defined by the equation $T(r, L)=T_{0}+\omega(r)+\sum_{i=1}^{M} c_{i} \xi_{i}(r)$.

Superscripts

, : Experimental value

: Estimated value.

\section{References}

[1] D. R. Reinhart, P. T. McCreanor, and T. G. Townsend, "The bioreactor landfill: its status and future," Waste Management and Research, vol. 20, no. 2, pp. 172-86, 2002.

[2] S. Renou, J. G. Givaudan, S. Poulain, F. Dirassouyan, and P. Moulin, "Landfill leachate treatment: review and opportunity," Journal of Hazardous Materials, vol. 150, no. 3, pp. 468-493, 2008.

[3] M. Zamorano, J. I. Pérez Pérez, I. Aguilar Pavés, and A. Ramos Ridao, "Study of the energy potential of the biogas produced by an urban waste landfill in Southern Spain," Renewable and Sustainable Energy Reviews, vol. 11, no. 5, pp. 909-922, 2007.

[4] N. J. Themelis and P. A. Ulloa, "Methane generation in landfill," Renewable Energy, vol. 32, pp. 12431257, 2007.

[5] A. Tikhonov and V. Arsenine, Méthodes de Résolution de Problémes mal Posés, Editions MIR, Moscow, Russia, 1976, Traduit du russe par Vladimir Kotliar.

[6] V. G. Dovi', A. P. Reverberi, and V. P. Meshalkin, "A general procedure for the estimation of diffusivities in solids," The Canadian Journal of Chemical Engineering, vol. 72, no. 6, pp. 1042-1046, 1994.

[7] R. B. Bird, W. E. Stewart, and E. N. Lightfoot, Transport Phenomena, John Wiley \& Sons, New York, NY, USA, 2002.

[8] H. S. Carslaw and J. C. Jaeger, Conduction of Heat in Solids, Clarendon Press, Oxford, UK, 2nd edition, 1959.

[9] R. Courant and D. Hilbert, Methods of Mathematical physics, Wiley-VCH, Berlin, Germany, 1989.

[10] V. A. Morozov, Methods for Solving Incorrectly Posed Problems, Springer, New York, NY, USA, 1984.

[11] ASHRAE, Ground Source Heat Pumps: Design of Geothermal Systems for Commercial and Institutional Buildings, American Society of Heating, Refrigeration and Air Conditioning Engineers, Atlanta, Ga, USA, 2008. 


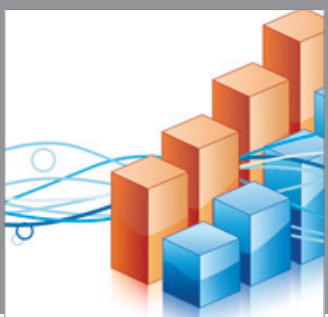

Advances in

Operations Research

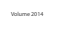

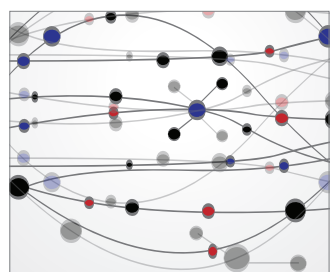

\section{The Scientific} World Journal
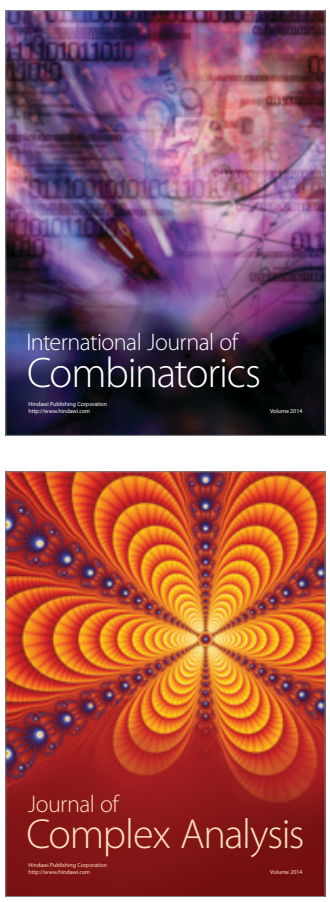

International Journal of

Mathematics and

Mathematical

Sciences
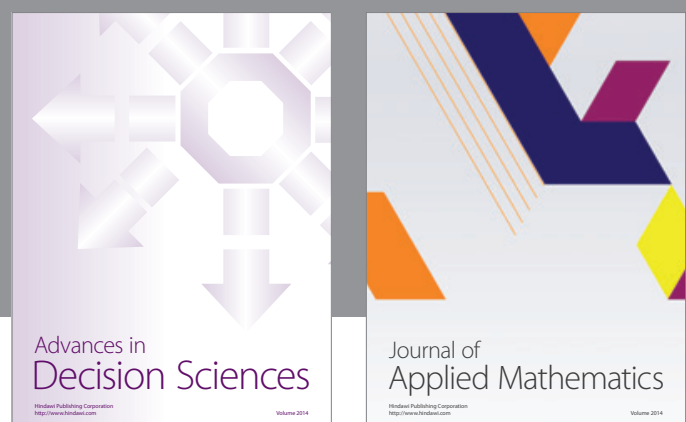

Journal of

Applied Mathematics
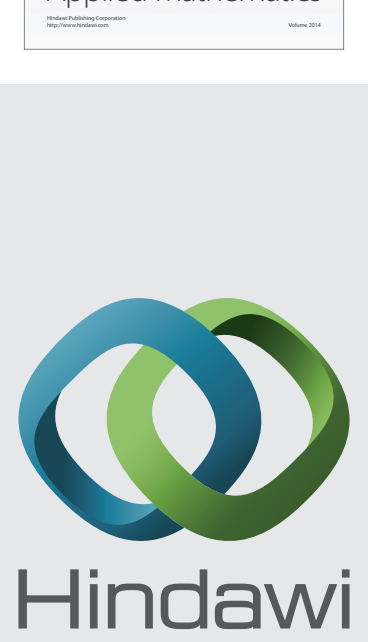

Submit your manuscripts at http://www.hindawi.com
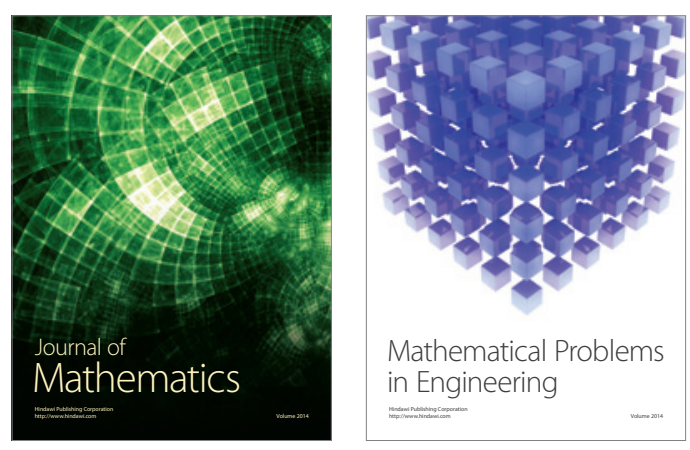

Mathematical Problems in Engineering
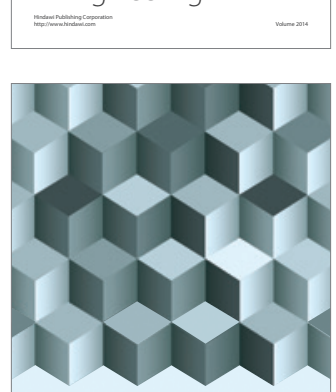

Journal of

Function Spaces
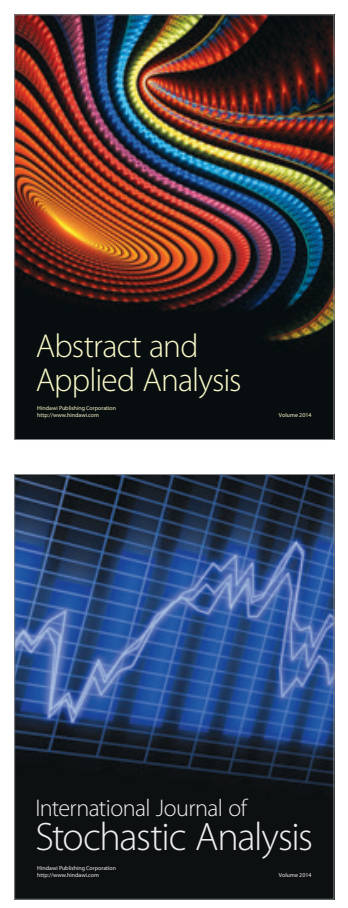

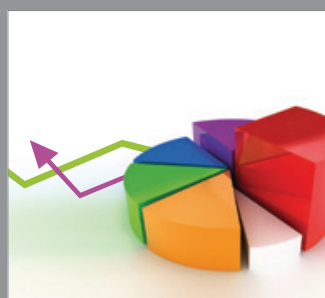

ournal of

Probability and Statistics

Promensencen
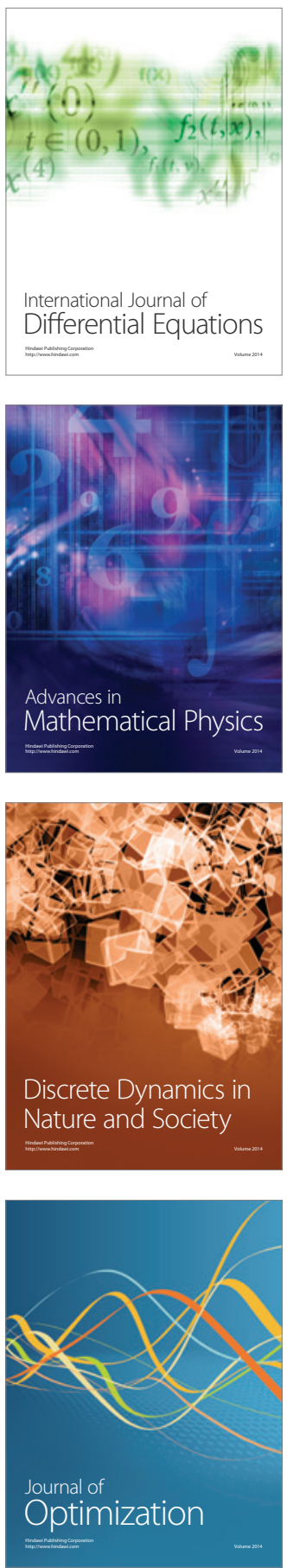